\title{
Measurement of the neutron energy spectrum of Back-n \#ES1 at CSNS
}

Yonghao Chen ${ }^{1,2}$, Guangyuan Luan ${ }^{3}$, Jie $\mathrm{Bao}^{3, *}$, Hantao Jing ${ }^{1,2}$, Qi An ${ }^{4,5}$, Huaiyong Bai ${ }^{6}$, Ping Cao ${ }^{4,5}$, Qiping Chen ${ }^{7}$, Pinjing Cheng ${ }^{8}$, Zengqi Cui ${ }^{6}$, Ruirui Fan $^{1,2,4}$, Changqing Feng ${ }^{4,5}$, Minhao $\mathrm{Gu}^{1,4}$, Fengqin Guo ${ }^{1,2}$, Changcai Han ${ }^{9}$, Zijie $\mathrm{Han}^{7}$, Guozhu $\mathrm{He}^{3}$, Yongcheng He ${ }^{1,2}$, Yuefeng $\mathrm{He}^{8}$, Hanxiong Huang ${ }^{3}$, Weiling Huang ${ }^{1,2}$, Xiru Huang ${ }^{4,5}$, Xiaolu Ji ${ }^{1,4}$, Xuyang $\mathrm{Ji}^{4,10}$, Haoyu Jiang ${ }^{6}$, Wei Jiang ${ }^{1,2}$, Ling Kang ${ }^{1,2}$, Mingtao Kang ${ }^{1,2}$, Bo $\mathrm{Li}^{1,2}$, Lun $\mathrm{Li}^{1,2}$, Qiang $\mathrm{Li}^{1,2}$, Xiao Li $^{1,2}$, Yang(a) $\mathrm{Li}^{1,4}$, Yang(b) $\mathrm{Li}^{1,2}$, Rong Liu ${ }^{7}$, Shubin Liu ${ }^{4,5}$, Xingyan Liu ${ }^{7}$, Yinglin $\mathrm{Ma}^{1,2}$, Changjun Ning ${ }^{1,2}$, Binbin $\mathrm{Qi}^{4,5}$, Jie Ren ${ }^{3}$, Xichao Ruan ${ }^{3}$, Zhaohui Song ${ }^{9}$, Hong Sun ${ }^{1,2}$, Xiaoyang Sun ${ }^{1,2}$, Zhijia Sun ${ }^{1,2,4}$, Zhixin Tan ${ }^{1,2}$, Hongqing Tang ${ }^{3}$, Jingyu Tang ${ }^{1,2}$, Pengcheng Wang ${ }^{1,2}$, Qi Wang ${ }^{3}$, Taofeng Wang ${ }^{11}$, Yanfeng Wang ${ }^{1,2}$, Zhaohui Wang ${ }^{3}$, Zheng Wang ${ }^{1,2}$, Jie $\mathrm{Wen}^{7}$, Zhongwei $\mathrm{Wen}^{7}$, Qingbiao $\mathrm{Wu}^{1,2}$, Xiaoguang $\mathrm{Wu}^{3}$, Xuan $\mathrm{Wu}^{1,2}$, Likun Xie ${ }^{4,10}$, Yiwei Yang ${ }^{7}$, Han $\mathrm{Yi}^{1,2}$, Li $\mathrm{Yu}^{1,2}$, Tao Yu ${ }^{4,5}$, Yongji $\mathrm{Yu}^{1,2}$, Guohui Zhang ${ }^{6}$, Jing Zhang ${ }^{1,2}$, Linhao Zhang ${ }^{1,2}$, Liying Zhang ${ }^{1,2}$, Qingming Zhang ${ }^{12}$, Qiwei Zhang $^{3}$, Xianpeng Zhang ${ }^{9}$, Yuliang Zhang ${ }^{1,2}$, Zhiyong Zhang ${ }^{4,5}$, Yingtan Zhao ${ }^{12}$, Liang Zhou ${ }^{1,2}$, Zuying Zhou ${ }^{3}$, Danyang $\mathrm{Zhu}^{4,5}$, Kejun $\mathrm{Zhu}{ }^{1,4}$, and Peng Zhu ${ }^{1,2}$

${ }^{1}$ Institute of High Energy Physics, Chinese Academy of Sciences (CAS), Beijing 100049, China

${ }^{2}$ Spallation Neutron Source Science Center, Dongguan 523803, China

${ }^{3}$ Key Laboratory of Nuclear Data, China Institute of Atomic Energy, Beijing 102413, China

${ }^{4}$ State Key Laboratory of Particle Detection and Electronics

${ }^{5}$ Department of Modern Physics, University of Science and Technology of China, Hefei 230026, China

${ }^{6}$ State Key Laboratory of Nuclear Physics and Technology, School of Physics, Peking University, Beijing 100871, China

${ }^{7}$ Institute of Nuclear Physics and Chemistry, China Academy of Engineering Physics, Mianyang 621900, China

${ }^{8}$ University of South China, Hengyang 421001, China

${ }^{9}$ Northwest Institute of Nuclear Technology, Xi' an 710024, China

${ }^{10}$ Department of Engineering and Applied Physics, University of Science and Technology of China, Hefei 230026, China

${ }^{11}$ School of Physics, Beihang University, Beijing 100083, China

${ }^{12} \mathrm{Xi}$ ' an Jiaotong University, Xi' an 710049, China

\begin{abstract}
The China spallation neutron source (CSNS) was built and started running since 2018. It produces neutrons by impinging $1.6 \mathrm{GeV}$ protons onto a tungsten target with $25 \mathrm{~Hz}$ repetition frequency. A beam line exploiting the back-streaming neutrons (Back-n) was built mainly for nuclear data measurement and started commissioning simultaneously with CSNS in 2018. There are two experimental endstations along the Back-n beam line: endstation 1 (\#ES1) with a neutron flight path of about $55 \mathrm{~m}$ and endstation 2 (\#ES2) with about $76 \mathrm{~m}$. The neutron energy spectra of both \#ES1 and \#ES2 were measured since it is important for feasibility study and analysis. In this paper, the measurement of the neutron energy spectrum of Back-n \#ES1 is reported. It is measured by a multi-layer fission chamber using the ${ }^{235} \mathrm{U}$ samples as the neutron converters. The neutron energy spectrum from $0.1 \mathrm{eV}$ to $30 \mathrm{MeV}$ is obtained. The integral neutron flux (from $0.1 \mathrm{eV}$ to $30 \mathrm{MeV}$ ) normalized to the proton beam power of $100 \mathrm{~kW}$ is $1.55 \times 10^{7}$ neutrons $/ \mathrm{cm}^{2} / \mathrm{s}$.
\end{abstract}

\section{Introduction}

The China Spallation Neutron Source (CSNS) is the first spallation neutron source in China. It is also the largest proton accelerator ever built in China up to now [13]. CSNS mainly supports the multidisciplinary research based on the neutron scattering technique [4, 5]. It is also capable of supporting multiple platforms, such as the application of high energy protons, muon beam and white neutron beam [6]. A beam line exploiting the backstreaming neutrons, named Back-n, is built mainly for the nuclear data measurements and started running simultaneously with CSNS in 2018 [7-10]. The neutron energy spectrum of Back-n is characterized since it is of great im-

*e-mail: baojie_ciae@126.com portance for feasibility study and analysis for the measurements. This paper presents the measurement of the neutron energy spectrum of Back-n endstation 1 (\#ES1) and reports the first results.

\section{Experimental setup}

\subsection{Back-n facility at CSNS}

CSNS is implemented by impinging $1.6 \mathrm{GeV}$ protons onto a tungsten target with $25 \mathrm{~Hz}$ repetition frequency. As fig. 1 shows, the incident proton beam is bent 15 degrees by a bending magnet before they bombard the tungsten target. The back-streaming neutrons pass through part of the proton tube and fly straight to reach the experimental endstations. The proton bending magnet can also sweep out 
the charged particles in the Back-n beam in the meantime. A cadmium plate is put at the neutron beam window to cut off the thermal neutrons. There are two endstations along the beam line: \#ES1 with a flight length of about $55 \mathrm{~m}$ and endstation 2 (\#ES2) with about $76 \mathrm{~m}$. Seven detector systems are arranged in different locations in two endstations [9]. They are: $\mathrm{C}_{6} \mathrm{D}_{6}$ detectors and a $4 \pi-\mathrm{BaF}_{2}$ array (GTAF-II) for neutron capture cross-section measurement, a multi-layer fast ionization chamber for fission cross-section measurement (FIXM), a total cross-section measurement spectrometer (NTOX), a light-charged particle emission detector array (LPDA), a detector array for prompt fission neutron spectrum measurements (FINDA) and a $4 \pi$ germanium detector array for gamma spectrum measurements (GAEA). The $\mathrm{C}_{6} \mathrm{D}_{6}$, FIXM, NTOX and LPDA have been used in day-one experiments and their first results will be published soon. The other three detector systems are under construction and will be available in a later phase.

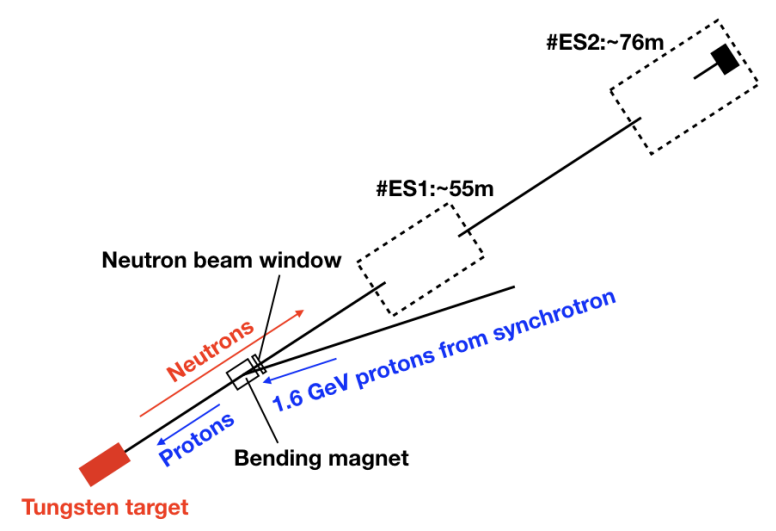

Figure 1: Layout of the Back-n beam line at CSNS.

The CSNS accelerator can be operated in doublebunch mode or single-bunch mode. Double-bunch mode means there are two bunches having same intensity and distribution in one proton pulse with a fixed time interval of $410 \mathrm{~ns}$. Single-bunch mode means only one bunch presents in one proton pulse. CSNS accelerator is generally operated in double-bunch mode since its beam intensity is twice higher and most of the other spectrometers are only using thermal neutrons where the double-bunch effect is negligible. However the single-bunch mode is applicable for some dedicated measurements. The measurement in this work is performed under double-bunch mode and the results are then corrected based on the simulation. More details is discussed in sect. 3.2

\subsection{MFC setup at \#ES1}

The detector used for measuring the neutron energy spectrum is a multi-layer fission chamber (MFC), in which three ${ }^{235} \mathrm{U}$ and one ${ }^{238} \mathrm{U}$ samples are mounted (fig. 2). The samples are electrodeposited on stainless steel backings. Their thicknesses are characterized by the $\alpha$ spectra. The electrodes are stainless steel foil as well. Each sample and the neighbor electrode form an independent cell. The

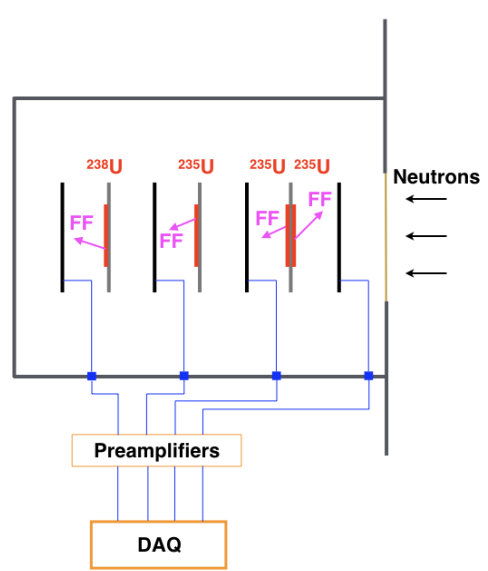

Figure 2: MFC setup at Back-n \#ES1.

chamber is filled with a mixture gas composed by $90 \%$ argon and $10 \%$ tetrafluoromethane and is sealed at atmospheric pressure. The gap between each sample and the electrode is $10 \mathrm{~mm}$ where a high voltage of about 300 volts is applied.

The sample backings are grounded and the signals are delivered by the electrodes. The signals pass through the preamplifiers and input the Back-n general-purpose Data Acquisition System (DAQ) [11]. The full waveforms of signals above the threshold are digitized with $1 \mathrm{GHz}$ sampling rate and 12-bit resolution. A dedicated routine is developed to analyze the signal pulse shapes offline to extract the interested information, such as the timing, amplitude, etc.

\section{Results and discussions}

\subsection{Time-of-Flight (TOF) method to determine neutron energy}

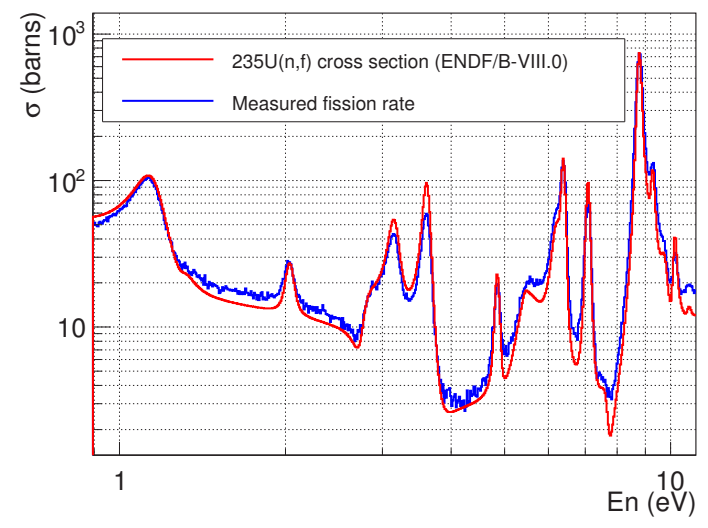

Figure 3: Comparison of the measured fission rate of ${ }^{235} \mathrm{U}$ and its fission cross-section from ENDF/B-VIII.0 [12] for calibrating the neutron flight path. 
To use the TOF method to determine the neutron energy, we first need to determine the flight path $L$ and starting flight time $T_{0} . L$ is calibrated by the resonance peaks of the fission rate of ${ }^{235} \mathrm{U}$ (fig. 3). A flight path is assumed and then adjusted until the measured fission rate match the database [12]. The determined flight path to the first ${ }^{235} \mathrm{U}$ sample is $55.67 \mathrm{~m}$. $T_{0}$ is calibrated by $\gamma$-flash signal, which is induced by the prompt $\gamma$-rays generated in the spallation process. Since the measurement is done in double-bunch mode, there are always two $\gamma$-flash signals as shown in fig. 4. We take the first one as the time reference for all the events and then correct it based on the simulation (more details in sec. 3.2). With a certain flight path $L$ and speed of light, we can define $T_{0}$ of every neutron pulse.

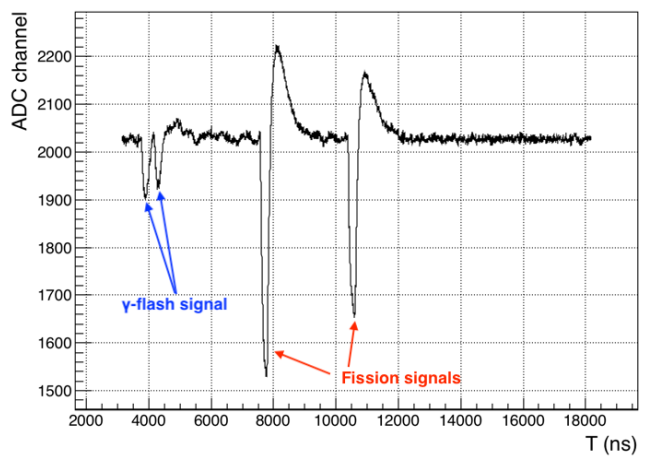

Figure 4: Signal frame including $\gamma$-flash signals and fission signals.
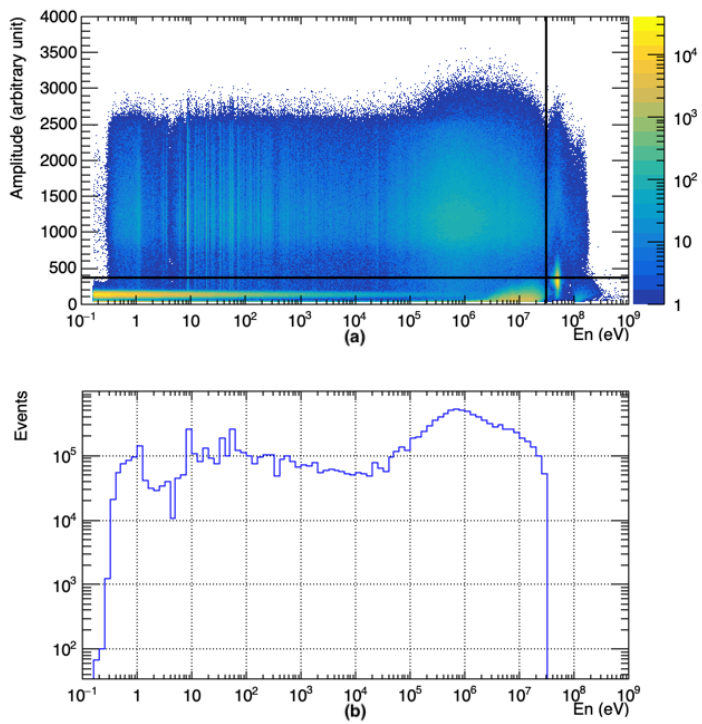

Figure 5: The 2D distribution of neutron energy versus signal amplitude (a). The fission rate as a function of neutron energy (b).

The 2-dimensional (2D) distribution of neutron energy versus signal amplitude is shown in fig. 5 (a). We set two cuts to extract the proper fission rate. The horizontal black solid line is the cut for the small amplitude events, which are mainly the $\alpha$ particles from sample decay but also some light charged particles induced by high energy neutrons. The vertical black solid line is set to cut the high energy events (above $30 \mathrm{MeV}$ ). Since the high energy region is intensively influenced by the second $\gamma$-flash. Figure 5 (b) shows the fission rate with 10 bins per decade (bpd) as a function of neutron energy after the application of the two cuts.

\subsection{Double-bunch correction}

Figure 5 (b) is the fission rate that measured in doublebunch mode. That is to say, the $T_{0}$ for all the events is referenced by the first proton bunch. Since the two proton bunches have same intensity and distribution and the time interval between them is well-defined (410 ns), the result of the double-bunch measurement is the direct overlap of two identical single bunch measurement with one bunch is delayed 410 ns. Furthermore, The neutron energy spectrum measured in \#ES2 with single-bunch mode verifies that the simulation can reproduce the experimental results well [13]. Therefore we correct the double-bunch to single-bunch based on the simulated results of \#ES1. The correction factor from $0.1 \mathrm{eV}$ to $30 \mathrm{MeV}$ shown in fig. 6 is multiplied to fig. 5 (b) to obtain the corrected fission rate.

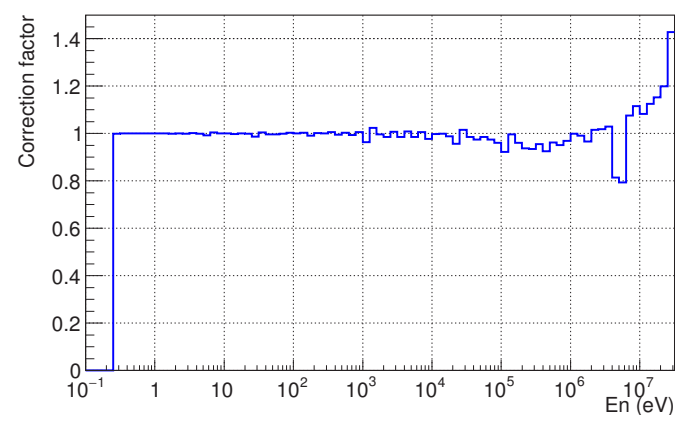

Figure 6: Correction factor for double-bunch mode as a function of neutron energy.

\subsection{Neutron flux}

The neutron energy spectrum, measured by a thin ${ }^{235} \mathrm{U}$ sample, can be calculated by

$$
\Phi\left(E_{n}\right)=\frac{R\left(E_{n}\right) K_{1}\left(E_{n}\right) K_{2}\left(E_{n}\right)}{\sigma\left(E_{n}\right) \varepsilon\left(E_{n}\right) N_{S}},
$$

where $\Phi\left(E_{n}\right)$ is the neutron flux inside an energy bin centered at energy $E_{n}, R\left(E_{n}\right)$ is the measured fission rate inside the same energy bin, $K_{1}\left(E_{n}\right)$ is the correction factor due to the double-bunch measurement as shown in fig. 6 , $K_{2}\left(E_{n}\right)$ is the uranium isotope correction since the ${ }^{235} \mathrm{U}$ is not highly enriched (more details can be found in reference [13]), $\sigma\left(E_{n}\right)$ is the neutron-induced fission cross section of ${ }^{235} \mathrm{U}$ that can be retrieved from ENDF/B-VIII.0 [12] (for 


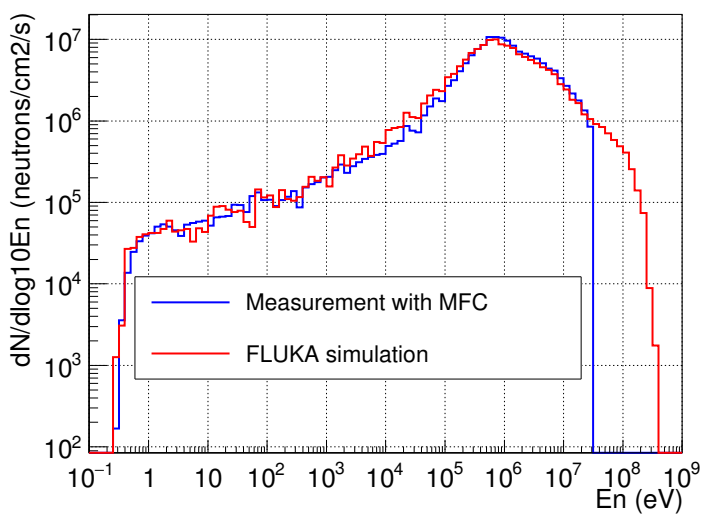

Figure 7: The comparison of the measured and simulated neutron energy spectrum of CSNS Back-n \#ES1 at $100 \mathrm{~kW}$ with 10 bpd with energy logarithmic scale.

data below $0.15 \mathrm{MeV}$ ) and IAEA standard references [14] (above $0.15 \mathrm{MeV}), \varepsilon\left(E_{n}\right)$ is the detection efficiency of fission fragment which is about $\sim 95 \%$ (more details in reference [13]), $N_{S}$ is the areal density (atoms/barn) of the ${ }^{235} \mathrm{U}$ sample.

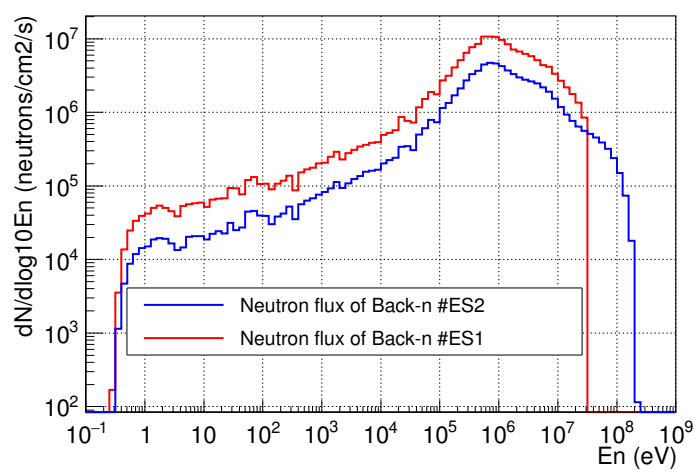

Figure 8: Comparison of the neutron energy spectrum of Back-n \#ES1 and \#ES2.

The results from $0.1 \mathrm{eV}$ to $30 \mathrm{MeV}$ normalized to the proton beam power of $100 \mathrm{~kW}$ is shown as the blue curve in fig. 7. The red curve is the results by FLUKA simulation [7] which agrees the measurement reasonably well. The comparison of the neutron energy spectrum between \#ES1 and \#ES2 is shown in fig. 8. The details of the measurement at \#ES2 is reported in references [13]. It can be seen that the spectra at \#ES1 and \#ES2 have similar shape but the flux at \#ES1 is higher due to the short flight path. Table 1 summarizes the fluxes of different energy range of both \#ES1 and \#ES2 at $100 \mathrm{~kW}$. The factor of the integral flux from $0.1 \mathrm{eV}$ to $10 \mathrm{MeV}$ between \#ES1 and \#ES2 is 2.28 .

\section{Conclusions and outlook}

This contribution reports the measurement of neutron energy spectrum of CSNS Back-n \#ES1 by the MFC. The measurement is performed under the double-bunch mode and is then corrected based on the simulation. The neutron energy spectrum from $0.1 \mathrm{eV}$ to $30 \mathrm{MeV}$ is obtained. The total flux from $0.1 \mathrm{eV}$ to $30 \mathrm{MeV}$ normalized to $100 \mathrm{~kW}$ beam power is $1.55 \times 10^{7}$ neutrons $/ \mathrm{cm}^{2} / \mathrm{s}$. The measured neutron energy spectrum at \#ES1 has a similar distribution with that of \#ES2 and the factor of integral neutron flux (from $0.1 \mathrm{eV}$ to $10 \mathrm{MeV}$ ) between them is 2.28 .

The spectrum at Back-n \#ES1 is preferred to be measured with single-bunch mode in the future to verify the present results. And it also should be measured based on the other reactions, such as ${ }^{6} \mathrm{Li}(\mathrm{n}, \mathrm{t})$ and ${ }^{10} \mathrm{~B}(\mathrm{n}, \alpha)$, to perform the cross check between different measurements and to finalize the results.

Table 1: Comparison of neutron fluxes of Back-n \#ES1 and \#ES2 in different neutron energy ranges at $100 \mathrm{~kW}$.

\begin{tabular}{ccc}
\hline Neutron energy range & \multicolumn{2}{c}{ Neutron fluxes (neutrons $/ \mathrm{cm}^{2} / \mathrm{s}$ ) } \\
\cline { 2 - 3 } & \#ES1 & \#ES2 \\
\hline $0.1-1 \mathrm{eV}$ & $1.15 \times 10^{4}$ & $4.08 \times 10^{3}$ \\
$1-10 \mathrm{eV}$ & $5.08 \times 10^{4}$ & $1.79 \times 10^{4}$ \\
$10-100 \mathrm{eV}$ & $8.75 \times 10^{4}$ & $3.01 \times 10^{4}$ \\
$0.1-1 \mathrm{keV}$ & $1.35 \times 10^{5}$ & $5.01 \times 10^{4}$ \\
$1-10 \mathrm{keV}$ & $3.05 \times 10^{5}$ & $1.23 \times 10^{5}$ \\
$10-100 \mathrm{keV}$ & $1.03 \times 10^{6}$ & $4.30 \times 10^{5}$ \\
$0.1-1 \mathrm{MeV}$ & $6.96 \times 10^{6}$ & $2.98 \times 10^{6}$ \\
$1-10 \mathrm{MeV}$ & $6.09 \times 10^{6}$ & $2.77 \times 10^{6}$ \\
$10-100 \mathrm{MeV}$ & - & $6.21 \times 10^{5}$ \\
$10-30 \mathrm{MeV}$ & $8.85 \times 10^{5}$ & - \\
\hline Total & $1.55 \times 10^{7}$ & $7.03 \times 10^{6}$ \\
\hline
\end{tabular}

\section{References}

[1] S. Fang et al., J. Kor. Phys. Soc. 48, 697 (2006).

[2] J. Wei et al., Nucl. Instrum. Meth. A 600, 10 (2009).

[3] H. Chen et al., Nat. Mater. 15, 689 (2016).

[4] Y. Xiao et al., Nano Energy, 49, 77 (2018).

[5] E. Zhao et al., Energy Storage Mater. 16, 354 (2019).

[6] J. Tang et al., Chin. Phys. C 34, 121 (2010).

[7] H. Jing et al., Nucl. Instrum. Meth. A 621, 91 (2010).

[8] H. Jing et al., Chin. Phys. C 37, 117002 (2013).

[9] Q. An et al., J. Instrum. 12, 07022 (2017).

[10] H. Yi et al., J. Instrum. 14, 02011 (2019).

[11] Q. Wang et al., Review of Scientific Instruments 89, 013511 (2018).

[12] D.A. Brown et al., Nucl. Data Sheets. 148, 1 (2018).

[13] Y. Chen et al., Eur. Phys. J. A 55, 115 (2019).

[14] A.D. Carlson et al., Nucl. Data Sheets 110, 3215 (2009). 